\title{
Freiwilliges Engagement - ein bildungsbiografischer Erfahrungsraum
}

\author{
Sascha Benedetti
}

Online publiziert: 11. März 2015

(c) Die Autor(en) 2015. Dieser Artikel ist auf Springerlink.com mit Open Access verfügbar.

Zusammenfassung Fortbestand und Weiterentwicklung moderner Gesellschaften sind nicht möglich ohne ein breites Spektrum gesellschaftlich verantwortlicher Tätigkeiten jenseits der Erwerbsarbeit, die unter dem Begriff „Freiwilliges Engagement“ zusammengefasst werden können. Unter Rückgriff auf empirisches Interviewmaterial skizziert der Beitrag das Spektrum und den Wandel der subjektiven bildungsbiografischen Bedeutungen freiwilligen Engagements aus der Perspektive der Engagierten. Dabei wird die besondere Eignung qualitativer Follow-up-Studien zur Erfassung eben jener längerfristigen bildungsbiografischen Wandlungsprozesse aufgezeigt. Nach einer systematisierenden Betrachtung bedeutsamer individueller sowie kollektiver Kontexte freiwilligen Engagements folgen abschließende Überlegungen zu dessen doppelter dynamischer Struktur.

Schlüsselwörter Freiwilliges Engagement · Bildungsprozesse im freiwilligen Engagement $\cdot$ Engagementforschung $\cdot$ Biographieforschung $\cdot$ Qualitative Längsschnittstudie

\begin{abstract}
The development of modern societies depends on a wide variety of an individual's social commitment beyond gainful employment that can be subsumed under the term "volunteering". Analysing empirical interviews, this article outlines the range and the transition of subjective educational biographic meanings of volunteering from the perspective of the volunteers. Thereby the appropriateness of qualitative longitudinal studies for specific research-purposes of long-term edu-
\end{abstract}

Dipl.-Päd. S. Benedetti ( $\square)$

Institut für Erziehungswissenschaft,

AG Allgemeine Erziehungswissenschaft,

Johannes Gutenberg Universität Mainz,

Mainz, Deutschland

E-Mail: benedett@uni-mainz.de 
cational biographic change processes can be revealed. According to a systematic consideration of significant individual and collective contexts of volunteering, there will be conclusive reflections about its double-dynamic structure.

Keywords Volunteering - Educational processes in volunteering Research on engagement · Research on biographies - Qualitative longitudinal studie

\section{Einleitung}

Freiwilliges Engagement ${ }^{1}$ ist in den vergangenen 30 Jahren verstärkt zum Gegenstand politischer, gesellschaftlicher sowie erziehungs- und sozialwissenschaftlicher Diskurse und Forschung geworden.

Das große gesellschaftspolitische Interesse am freiwilligen Engagement wird in vielfacher Weise erkennbar: Auf nationaler sowie internationaler Ebene wurden seit den 1980er Jahren eine Reihe von Aktions- und Gedenktagen und -jahren - z. B. das „Europäische Jahr der Freiwilligentätigkeit“ (2011) - ausgerufen. Der seit 1999 im Auftrag der Bundesregierung in Fünfjahresintervallen erhobene, repräsentativ angelegte Freiwilligensurvey (von Rosenbladt 2001; Gensicke et al. 2006) kann ebenso als deutlicher Indikator für die politisch anerkannte Bedeutsamkeit des freiwilligen Engagements angesehen werden, wie die 1999 durch den Bundestag erfolgte Einsetzung der Enquete-Kommission „Zukunft des bürgerschaftlichen Engagements“, deren Abschlussbericht eine Bestandsaufnahme sowie eine Vielzahl von Vorschlägen zur weiteren Steigerung des bürgerschaftlichen Engagements beinhaltet (EnqueteKommission 2002). Auch das 2002 gegründete „Bundesnetzwerk Bürgerschaftliches Engagement" ist Ausdruck der allgemein gestiegenen Anerkennung des freiwilligen Engagements sowie des gesteigerten Selbstbewusstseins der Engagierten.

In den erziehungs- und sozialwissenschaftlichen Diskursen nimmt die Auseinandersetzung mit dem komplexen Thema des freiwilligen Engagements, insbesondere hinsichtlich seiner Prämissen, Implikationen und Konsequenzen, mittlerweile einen nicht unerheblichen Stellenwert ein. Dies zeigt sich nicht nur daran, dass das freiwillige Engagement in einer ganzen Reihe unterschiedlicher Diskurslinien (Braun 2000; Priller 2010) - wie z. B. dem Diskurs um die Krise des Sozialstaats oder des Wertewandels - aufgegriffen wird, sondern auch daran, dass in Fachzeitschriften mittlerweile ganze Hefte zum freiwilligen oder bürgerschaftlichen Engagement (z. B. Zeitschrift für Erziehungswissenschaft 2/2010) erschienen sind. Ferner kann auch das 2011 herausgegebene erste Handbuch Bürgerschaftliches Engagement (Olk und

\footnotetext{
${ }^{1}$ Obwohl auf nationaler sowie internationaler Ebene eine Vielzahl graduell unterschiedlich konnotierter Termini (gesellschaftliches Engagement, bürgerschaftliches Engagement, soziales Engagement, Freiwilligenarbeit, Ehrenamt, Volunteering etc.) für das freiwillige und nicht auf einen persönlichen materiellen Gewinn ausgerichtete Handeln zum Wohle anderer oder der Gemeinschaft kursiert, liegt all diesen Begriffen ein grundsätzliches Engagementverständnis zugrunde, welches durch Freiwilligkeit, Unentgeltlichkeit, Gemeinwohlorientierung, Öffentlichkeitsbezug und kooperatives/gemeinschaftliches Handeln gekennzeichnet ist (Enquete-Kommission 2002, S. 86 ff.; Priller 2010, S. 198). Auch für den im vorliegenden Beitrag verwendeten Terminus des freiwilligen Engagements sind diese fünf Merkmale charakteristisch, jedoch umfasst er im Gegensatz zum Begriff des Ehrenamts auch neuere Engagementfelder, Engagementsettings und Engagementformen (Jakob 2001, S. 167) sowie kurzfristiges oder punktuelles Engagement.
} 
Hartnuß 2011) als Hinweis auf das große theoretische sowie empirische Interesse am Engagement betrachtet werden.

Das enorme empirische Interesse am freiwilligen Engagement wird keineswegs nur in den groß angelegten und primär quantitativ ausgerichteten Untersuchungen, wie etwa dem Freiwilligensurvey sichtbar, sondern auch in der Vielzahl kleinerer qualitativer sowie quantitativer Studien, welche entweder einzelne Engagementfelder (z. B. Vereine oder Unternehmen), spezifische Personengruppen (z. B. Senioren, Jugendliche, Migranten) oder konkrete empirische Fragen (Motive für die Ausübung freiwilligen Engagements) in den Blick nehmen. Innerhalb der Engagementforschung sind in den vergangenen zehn Jahren die individuellen Lern-, Bildungs- und Kompetenzerwerbsprozesse der Engagierten verstärkt zum Forschungsgegenstand geworden (Düx et al. 2008; Hansen 2008; Hübner 2010). ${ }^{2}$ Beispielsweise haben Düx et al. (2008) in ihrer Studie ${ }^{3}$ herausgearbeitet, dass Jugendliche im freiwilligen Engagement vielfältige Lern- und Bildungserfahrungen sammeln und dabei ein breites Spektrum an sozialen, personalen, politischen, fachlichen, organisationalen, medialen, praktischen Kompetenzen etc. erwerben können, welche ihrerseits wiederum in anderen Lebensbereichen - beispielsweise der Erwerbsarbeit - genutzt werden können. Empirisch bisher noch eher unerschlossen sind allerdings die längerfristigen (Aus-)Wirkungen der Aneignungsprozesse - und damit verbunden der bildungsbiografischen Bedeutungen - des freiwilligen Engagements aus der Perspektive der Engagierten. Da die längerfristige bildungsbiografische Bedeutung des Engagements durch Einmalbefragungen gegenwärtig oder ehemals Engagierter nicht ausreichend greifbar wird, sind zu ihrer empirischen Erfassung Längsschnittstudien erforderlich (Priller 2010, S. 208).

Der vorliegende Artikel versucht aus einer bildungsbiografietheoretischen Perspektive einen Beitrag zur Beantwortung der oben aufgeworfenen Frage nach dem Wandel der subjektiven bildungsbiografischen Bedeutung freiwilligen Engagements $\mathrm{zu}$ leisten. Zunächst werden bildungs- und biografietheoretische Vorüberlegungen skizziert und daraus abgeleitet, warum qualitative Follow-up-Studien einen Zugang zum Wandel der subjektiven bildungsbiografischen Bedeutung freiwilligen Engagements darstellen (Kap. 2). Aus dieser Perspektive wird unter Rückgriff auf das Interviewmaterial einer qualitativen Follow-up-Studie zunächst an drei Interviews das Spektrum der bildungsbiografischen Bedeutung des freiwilligen Engagements aus der Perspektive der Engagierten entfaltet (Kap. 3). Anschließend wird an einem dieser drei Fälle exemplarisch der Wandel des Engagements sowie dessen subjektiver Bedeutung skizziert (Kap. 4). Nach einer knappen Systematisierung der Kontextualität des freiwilligen Engagements (Kap. 5) erfolgen abschließende Überlegungen zur „doppelten dynamischen Struktur“" des freiwilligen Engagements (Kap. 6).

\footnotetext{
${ }^{2} \mathrm{Zu}$ den Möglichkeiten und Grenzen des Transfers des im Engagement Angeeigneten in die Erwerbsarbeit vgl. Söker und Mutz (2003).

${ }^{3} \mathrm{Im}$ Rahmen der Studie wurden mit 74 engagierten Jugendlichen und 13 ehemals engagierten Erwachsenen leitfadengestützte Face-to-Face-Interviews geführt. Zudem fand eine standardisierte retrospektive Vergleichsbefragung mit 1.500 ehemals engagierten Erwachsenen sowie 552 in ihrer Jugend nicht engagierten Erwachsenen statt.
} 


\section{Ziele und Gegenwartsindexikalität von Bildung und Biografien}

Dem humboldtschen Begriffsverständnis folgend, zielt die Bildung des Menschen darauf ab, ,soviel Welt als möglich zu ergreifen, und so eng als er nur kann, mit sich zu verbinden“ (Humboldt 1960 [1793], S. 235). Bildung kann somit als die intensive „Verknüpfung unseres Ichs mit der Welt“ (ebd.) bzw. die „weitest mögliche ,Aneignung" von Welt" (Luhmann 2002, S. 188) verstanden werden. Da Individuen dieser sich von ihnen anzueignenden Welt allerdings keineswegs völlig unverbunden gegenüberstehen, ist Bildung kein ,subjektinterner Prozess“ (Fischer et al. 2010, S. 253), sondern vielmehr die Aneignung und Konstitution von Welt eines sich bereits in einer jeweils spezifischen individuellen Position in der Welt befindlichen Subjekts. In seiner Reformulierung des humboldtschen Bildungsbegriffs weist Kade (2012) darauf hin, dass diese Aneignung und Konstitution von Welt in modernen Gesellschaften keineswegs mehr nur auf die Aufhebung von Fremdheit und die bloße Übernahme gegenwärtiger Gesellschaftsverhältnisse reduziert sei. Stattdessen ziele Bildung auf die „Befreiung von sozialen und biografischen Einengungen und Fremdbestimmungen des Lebens" (Kade 2012, S. 39) ab. Autonomie bzw. Selbstbestimmung stellt damit ein zentrales, jedoch keineswegs das einzige Ziel von Bildung dar. Als ein zweites Ziel von Bildung und Bildungsprozessen kann gerade vor dem Hintergrund der Diskussionen um die reflexive Moderne das Moment der Authentizität betrachtet werden, welches zwar mit dem Motiv der Autonomie verwoben, jedoch anders akzentuiert ist. Während Autonomie als Bildungsziel das Streben des Subjekts nach Emanzipation und damit nach Selbstbehauptung gegenüber gesellschaftlicher Fremdbestimmung hervorhebt, betont das Bildungsziel der Authentizität das Streben des Subjekts nach Selbstverwirklichung und Selbstfindung. Als drittes bedeutsames Bildungsziel kann das Motiv der Anerkennung ${ }^{4}$ betrachtet werden, welches weder die Identitätsfindung/-entfaltung noch die Behauptung dieser Identität gegenüber gesellschaftlichen Vereinnahmungen, sondern stattdessen das Streben des Subjekts nach dem Erleben von Wertschätzung seiner Individualität durch einzelne Personen, bestimmte Gruppen oder die Gesellschaft ins Zentrum rückt. Diese drei Bildungsziele tragen in ihrem Zusammenspiel maßgeblich dazu bei, das „Grundversprechen der Moderne“ (ebd., S. 40) einzulösen, indem sie das Subjekt dazu befähigen ,ein selbstbestimmtes Leben nach eigenen Maßstäben führen zu können“ (ebd.).

Da Bildung sich in einem Gefüge aus stetig wandelnden kollektiven und individuellen Kontexten vollzieht, ist sie durch ein hohes Maß an Prozessualität und Dynamik gekennzeichnet. In biografischen Erzählungen findet die Versprachlichung der vom Bildungssubjekt hergestellten Verknüpfung der von ihm gegenwärtig als bedeutsam empfundenen Lebensereignisse statt. Biografien und biografische Erzählungen sind immer Bildungsbiografien, denn die in ihnen thematisierten Lebenslaufereignisse und Lebenslauferfahrungen sowie deren Aneignung sind stets auch Bildungsereignisse, welche sich in einem sich stetig wandelnden Gefüge individueller und kollektiver Kontexte bewegen. Da sich dieses Kontextgefüge und mit ihm auch das Bildungssubjekt selbst sowie dessen Deutung und Strukturierung seiner Lebenserfahrungen im Zeitverlauf stetig wandeln, haben bildungsbiografische Erzählungen stets einen

\footnotetext{
${ }^{4}$ Zum Anerkennungsbegriff vgl. insbesondere Honneth (1992).
} 
„Gegenwartsindex“ (Hof et al. 2010, S. 331). Aufgrund dieser kontextgebundenen Zeitindexikalität haben Bildungsbiografien den Charakter von jeweils gegenwartsbezogenen „Momentaufnahmen“ (ebd.) in „Serien von Bildungsgestalten“ (ebd.) im Sinne von ,verstetigte[n; S.B.] Formen lernbezogener, aber nicht lernzentrierter, individueller Aneignung von Welt" (Kade et al. 2008, S. 11). Das Theorem der Serialität von Bildungsbiografien macht deutlich, dass der Wandel von Bildungsbiografien lediglich durch die Relation mehrerer biografischer Erzählungen derselben Person erfasst werden kann. Denn in jeder biografischen Erzählung trifft der Interviewte eine (interview-)gegenwartsspezifische Auswahl aus dem Gesamt seiner bisherigen Lebenslaufereignisse, welche er wiederum in ebenfalls gegenwartsspezifischer Weise beschreibt und verknüpft. Erst durch den Vergleich der in den Interviews thematisierten Lebenslaufereignisse und der Beschreibungsmuster (Kade und Hof 2010) wird der Wandel von Bildungsbiografien empirisch greifbar.

\section{Die bildungsbiografische Bedeutung des freiwilligen Engagements}

An die im vorangegangenen Abschnitt skizzierten Überlegungen anknüpfend, wird im Folgenden auf der Basis von drei Interviews ${ }^{5}$ aufgezeigt, dass Autonomie, Anerkennung und Authentizität keineswegs rein abstrakt-theoretische Bildungsziele sind, sondern drei Pole eines empirisch vorzufindenden Spektrums bildungsbiografischer Bedeutungen freiwilligen Engagements darstellen. Die hierzu ausgewählten Interviews entstammen dem Korpus des von der DFG geförderten Projekts „Prekäre Kontinuitäten. Der Wandel von Bildungsgestalten im großstädtischen Raum unter den Bedingungen der forcierten Durchsetzung des Lebenslangen Lernens“. Im Rahmen des Projekts wurden jeweils zwei bildungsbiografisch akzentuierte Interviews im zeitlichen Abstand von 25 Jahren (1983/1984 und 2008/2009) mit Erwachsenen, welche sich zum Zeitpunkt des ersten Interviews als Teilnehmende oder Lehrende an Volkshochschulen bewegten, geführt. Während der Fragefokus der ersten Interviewwelle primär auf den Lehr- und Lernerfahrungen im Kontext der Volkshochschule lag, stand in der zweiten Interviewwelle eher das Lebenslange Lernen im Zentrum. ${ }^{6}$ Bei den drei folgenden Fällen haben die Interviewten in mindestens einer ihrer beiden bildungsbiografischen Erzählungen ein aktuelles oder aber vergangenes freiwilliges Engagement als biografisch bedeutsam thematisiert.

\footnotetext{
${ }_{5}^{5}$ Die Nummerierung entstammt dem Sample der 85 Interviews umfassenden ersten Erhebungswelle.

${ }^{6}$ Die Analyse der Interviews vollzog sich in folgenden Schritten: 1) Erstellung umfangreicher, tabellarischer Timelines. Hier wurden die in den beiden Interviews eines Falles thematisierten objektiven Lebenslaufdaten festgehalten und ihnen wurden diejenigen Interviewstellen, in denen die Interviewten auf diese Daten zu sprechen kommen, zugeordnet. 2) Kontrastierende Rekonstruktion der jeweils spezifischen Verläufe der bildungsbiografischen Stationen in den beiden Interviews eines Falles. 3) Rekonstruktion und Relation der beiden jeweils (interview-)gegenwartsbezogenen Bildungsgestalten eines Falles. Das methodische Vorgehen im Rahmen des Projekts orientierte sich an dem explorativ-interpretativen Grundprinzip einer engen Verknüpfung von empirischer Forschung und Theorieentwicklung (Strauss 1991), der Unterscheidung von formulierenden und reflektierenden Analyseschritten sowie der Einbettung der Einzelfallrekonstruktionen in übergreifende Vergleichshorizonte (Nohl 2008). Zum methodischen Vorgehen vgl. Fischer und Kade (2012).
} 


\subsection{TN 82: Freiwilliges Engagement als Ort des autonomen Handelns}

TN 82 ist männlich, zum Zeitpunkt des ersten Interviews (1984) 28 Jahre alt, hat Ingenieurwesen studiert, arbeitet in der Datenverarbeitung einer Firma und engagiert sich seit zwei Jahren in einem Arbeitskreis, welcher sich die ökologische Aufklärung der Öffentlichkeit zum Ziel gesetzt hat. Zudem ist er in der Friedensbewegung aktiv.

TN 82 erzählt, dass er angeregt durch die in den 1980er Jahren entbrannte gesellschaftliche „Anti-Atomkraftdiskussion“ (I1/S. 4/Z.3) einen VHS-Kurs zum Thema Energie besucht habe, um ,mal ein bißchen mehr auf die Theorie zu gehen, mal gucken, was es für Alternativen gibt" (1/4/4 f.). Anschließend sei er auf den VHSKurs „Ökologie. Anstoß zum neuen Denken“ aufmerksam geworden und habe auch diesen Kurs besucht, in welchem die Vermittlung von ökologischen Inhalten „locker gehandhabt“ (1/3/19) bzw. ,halt so spielerisch“(1/3/19 f.) ablief. Auf die im Kurs vermittelten Inhalte geht TN 82 nicht ein, sondern berichtet lediglich, dass er sich zwar schon im Vorfeld für das Thema Ökologie interessiert, jedoch vor dem Kursbesuch „noch nie tiefer reingeguckt“ (1/3/21) habe. Angeregt durch die während des Kurses gesammelten Erkenntnisse kam er gegen Ende des Kurses zu dem Schluss, „man müßte weitermachen“ (1/3/24) und wurde daraufhin vom Kursleiter zur Mitarbeit in dessen - institutionell ebenfalls von der VHS gerahmten - ökologischen Arbeitskreis eingeladen. TN 82 ist „dann damals auch hingegangen“(1/3/19) - jedoch nicht ohne sich vorher auch die Arbeitskreisbeschreibung im Kursprogramm anzusehen. Hatte er die beiden vorangegangenen Kurse aus einem primär theoretischen Interesse heraus besucht, so sprach ihn an der Ausschreibung des Arbeitskreises insbesondere an, „daß man eben auch nicht nur Theorie macht, sondern auch mal praktische Sachen zusammen macht“" (1/3/32 f.). Im Rahmen des ökologischen Arbeitskreises bildet die gemeinsame, nicht-leiterzentrierte Erschließung von ökologischen Problemstellungen die Basis für die darauf aufbauende Konzeptionierung, Entwicklung und Herstellung von Aufklärungsartefakten (Plakate, Broschüren etc.), welche TN 82 und die anderen Arbeitskreismitglieder dann - z. B. im Rahmen von Ausstellungen - der Öffentlichkeit präsentieren, um diese für ökologische Fragestellungen zu sensibilisieren. Der Weg ins freiwillige Engagement stellt sich bei TN 82 somit als ein sich über mehrere Stationen hinweg vollziehender Prozess dar, an dessen Anfang ein thematisches Interesse an ökologischen Fragestellungen und ein Bedürfnis nach dem Erwerb von entsprechendem Wissen stand. Seine politische Aufklärung vollzieht sich in einem Dreischritt von der Aufklärung durch bereits Aufgeklärte (Kurs 1) über die eher spielerische Auseinandersetzung mit Inhalten unter Anleitung von bereits Aufgeklärten (Kurs 2) bis hin zur nicht-leitungszentrierten Verbindung von gemeinsamer theoretischer Auseinandersetzung mit ökologischen Inhalten mit informativaufklärerischem Handeln in der Öffentlichkeit (Arbeitskreis).

TN 82 hebt in der Beschreibung seines Engagements im Arbeitskreis dessen besondere Struktur hervor. Erstens tritt der Leiter des Arbeitskreises in der Wahrnehmung von TN 82 weniger in einer dominierenden „Führungsrolle“ (1/19/25), sondern vielmehr als ,gleichwertiger Teilnehmer“ (1/19/8) auf und lässt den Arbeitskreismitgliedern damit - trotz seines Wissensvorsprungs - viel Raum zur Partizipation und Gestaltung des Geschehens. Zweitens betont TN 82, dass die „eigentliche Arbeit““ $(1 / 20 / 31)$ in Form der vertiefenden inhaltlichen Auseinandersetzung sowie der Kon- 
zeption und Vorbereitung der Aufklärungsaktivitäten nicht in den wöchentlichen Treffen des Arbeitskreises, sondern in den ausgelagerten Treffen unter weitgehender Zurückhaltung oder gar Abwesenheit des Leiters stattfindet. Drittens hat TN 82 beim Engagement im Arbeitskreis das Gefühl, ,daß ich was tun konnte, das war nicht einfach nur was reden oder lesen oder was, sondern was tun“ (1/6/7 ff.). Im ökologischen Engagement eröffnet sich TN 82 ein Raum zum aktiven aufklärerischen Handeln jenseits einer klar hierarchisch aufgebauten Struktur und damit zum relativ autonomen Agieren.

Die zentrale Bedeutung des freiwilligen Engagements als Möglichkeitsraum für das von TN 82 angestrebte autonome Handeln offenbart sich erst durch die Einbeziehung seiner Ausführungen zur eigenen Berufssituation. Er erzählt, dass ihm seine Arbeit in der Datenverarbeitung durchaus ,auch irgendwie Spaß“ (1/27/33) mache, aber er „stehe halt nicht hinter den Computern, die ich baue“ (1/27/21 f.). Zudem arbeite er in einer „hierarchischen gewachsenen Firma“ (1/22/13), in der er zwar in einem Team arbeite, das gegenüber der Leitung als gleichberechtigte „Gruppe immer auftreten“ (1/22/11 f.) würde, dessen Autonomie (und damit die Autonomie von TN 82) aber bei der Entscheidung über den Verwendungszweck der konstruierten PCs endet, denn er habe ,überhaupt keinen Einfluss darauf, wo die Sachen eingesetzt werden" ( $(1 / 28 / 1 \mathrm{f}$.).

Das freiwillige Engagement stellt somit für TN 82 einen bildungsbiografisch bedeutsamen Erfahrungsraum dar, da er dort Möglichkeiten zum autonomen Denken, Handeln und Entscheiden gefunden hat, welche ihm im Kontext der Erwerbsarbeit fehlen. Aufgrund dieser Konstellation misst TN 82 diesem Engagement zum Zeitpunkt des Interviews eine größere persönliche Bedeutung zu als seiner Erwerbstätigkeit, was sich auch daran zeigt, dass er seine Arbeitszeit reduziert hat. Zwar sieht TN 82 in seinem Engagement weit mehr Möglichkeiten zum autonomen Handeln als in der Erwerbsarbeit, jedoch betrachtet er auch die Möglichkeiten des autonomen Handelns im Arbeitskreis aufgrund dessen institutioneller Angebundenheit an die VHS als limitiert und damit mittelfristig für sich als unzureichend. Auch wenn das bisherige Engagement als „Einstieg“ (1/16/13) für ihn wichtig war, will er Zeit und Energie zukünftig weniger in dieses Engagement, sondern mehr in stärker politisierte und vor allem offensiver in der Öffentlichkeit agierende Engagementformen investieren. Ihm schwebt ein Engagement „mehr schon so in autonomer Richtung“ (1/14/13 f.) vor, um ,so direkte Aktionen zu machen, also eher Beispiele zu machen als zu reden" (1/14/22). Als Beispiel verweist er auf sein Engagement in der Friedensbewegung in Form des außerparlamentarischen Protestes, bei dem er auch mit dem Gesetz in Konflikt geraten und infolgedessen „verknackt worden[sei; S.B.] wegen Nötigung" (1/14/16 f.). Diese Überlegungen von TN 82 weisen auf den temporären und dynamischen Charakter des freiwilligen Engagements und seiner Bedeutung für die Engagierten hin.

Gedanklich befindet sich TN 82 zum Zeitpunkt des Interviews aber keineswegs nur hinsichtlich seines Engagements, sondern auch beruflich in einer Phase der auf Steigerung der Handlungs- und Entscheidungsautonomie gegenüber institutioneller oder personaler Fremdbestimmung abzielenden Neu- und Umorientierung. Er berichtet von der noch eher vagen Idee, mit Gleichgesinnten einen „alternativen Betrieb“ (1/28/3) zu gründen, um sich dort „schon ein bißchen besser verwirklichen 
zu können“" (1/28/4 f.) als in seinem gegenwärtigen Angestelltenverhältnis. Die Überlegungen über mögliche Veränderungen der Erwerbsarbeitssituation zeigen auf, dass die bildungsbiografische Bedeutung des freiwilligen Engagements für TN 82 keineswegs darauf beschränkt ist, ihm einen Erfahrungsraum zum Erleben von Autonomie und damit ein Gegengewicht zur aktuellen Erwerbsarbeitssituation zu eröffnen. Vielmehr wirken die Erfahrungen und Aneignungsprozesse im Engagement auch als Impulsgeber für Überlegungen über eine berufliche Veränderung. Ob und wie diese berufliche Veränderung in der Zukunft tatsächlich vollzogen wird, ist zum Zeitpunkt des ersten Interviews noch offen.

\subsection{TN 72: Freiwilliges Engagement als Ort der Selbstfindung und Selbstverwirklichung}

TN 72 ist männlich, zum Zeitpunkt des ersten Interviews (1984) 38 Jahre alt, hat nach einer Tätigkeit im Industriesektor BWL studiert und arbeitet nun in der EDVOrganisation einer Anstalt des öffentlichen Rechts. Er engagiert sich seit drei Jahren im selben ökologischen Arbeitskreis wie TN 82 und ist zudem in einer Lebensmittelkooperative aktiv.

Auch TN 72 berichtet, nicht auf direktem Wege ins Engagement im Arbeitskreis gelangt zu sein. Am Ende seines BWL-Studiums bekam er ,bißerl Schwierigkeiten“ (I1/S. 1/Z.13), da sich die ökonomischen Studieninhalte ,nicht mehr so ganz vertragen“ (1/1/14 f.) hätten mit seinem, im Zuge der gesellschaftlichen Aufklärung entstandenen, ökologischen Bewusstsein. Ökologisches Bewusstsein und Ökonomiestudium respektive berufliche Identität stellten für ihn einen scheinbar unüberbrückbaren Widerspruch dar, woraufhin er die „Lust“ (1/1/17) am Studium verlor, ,nichts mehr gemacht habe und das nur noch über die Bühne gebracht habe, so gerade schlecht und recht" (1/1/17 ff.). Der innere Konflikt zwischen Ökonomie und Ökologie beeinflusste seine Entscheidung für eine Arbeitsstelle nach dem Studium. Er lehnte das Angebot eines Chemiekonzerns ab, denn ,das wäre mir bißchen zu schwierig geworden, da hätte ich ein bißerl Schwierigkeiten mit mir selber bekommen“ (1/7/33 f.) und trat eine Stelle in der Datenverarbeitung einer Anstalt des öffentlichen Rechts an. Das gegen Ende des Studiums aufkommende diffuse „Unbehagen“ (1/1/12) blieb aber bestehen und wurde für TN 72 zum Anlass, einen VHS-Kurs, dessen Titel „Unbehagen mit der Umwelt“ exakt sein damaliges Empfinden beschreibt, in der Hoffnung zu besuchen, „vielleicht ein bißchen was in Angriff nehmen“ (1/1/23 f.) zu können und „das Unbehagen da ein bißerl anzupacken“ (1/1/24 f.). Dort erhielt er ein Überblickswissen über ökologische Zusammenhänge und damit einen „Einstieg“ (1/3/1). Danach besuchte er kurzzeitig ein Diskussionsforum, brach die Teilnahme aber ab, da dort „bloß diskutiert“ (1/1/28 f.) bzw. „bloß große Reden geführt" (1/1/29) wurden, während er aber nach dem erfolgten inhaltlichen Einstieg nun ,konkret was machen“ (1/1/30) bzw. ,gerne eben schon was umsetzen“ (1/3/12) wollte. Auf der Suche nach einer Möglichkeit, sich zu engagieren, stieß er auf den ökologischen Arbeitskreis, in dessen Ausschreibungstext ,stand was von Ausstellungen drin, praktischen Arbeiten und so und einfach von konkreten Dingen" (1/1/32 ff.). Da diese Beschreibung seinem Wunsch nach ökologischem Aktivwerden entsprach, wurde er Mitglied dieses Arbeitskreises. 
In den Ausführungen zu seinem Engagement im Arbeitskreis hebt TN 72 hervor, dass er hier eine Möglichkeit fand, ,,mit seinem eigenen Unbehagen mit der Situation überhaupt mit der Umweltsituation und auch meine, mein Unwissen, mit der Situation fertig zu werden“ (1/1/12) und bereits auf mehreren Ebenen ,eine persönliche Entwicklung durchgemacht“" (1/4/20 f.) habe. Erstens konnte er sich ökologisches Wissen sowie einen kritischeren Blick auf ökologische Zusammenhänge aneignen und auf der Basis dieses Wissens teilweise seine Lebenspraxis außerhalb des Arbeitskreises (z. B. seine Ernährungsgewohnheiten) umstellen. Zweitens erklärt er, dass er im Arbeitskreis eine größere Selbst- und Handlungssicherheit aufbauen konnte. Hatte er ,vielleicht auch einfach persönlich bedingt“ (1/7/23 f.) zu Beginn seines Engagements im Arbeitskreis „ein bißerl Hemmungen (...) da mich einzubringen“ (1/7/24), so kann er sich nun ,ein kleines bißchen freier und offener bewegen“" (1/7/22). Diesen Gewinn an Selbstsicherheit führt er auf die Arbeitskreismitglieder zurück, welche „freier und offener miteinander umgehen“" (1/13/26) als die Menschen, denen er in anderen Lebenszusammenhängen begegnet. Daher bezeichnet er die im Arbeitskreis Engagierten als ,andere[n; S.B.] Typ Mensch“ (1/4/29 f.). Im gemeinschaftlichen Engagement findet TN 72 in den Arbeitskreismitgliedern Menschen, die ihm in einer Phase der umfassenden Verunsicherung hinsichtlich des Umgangs mit sich selbst, mit anderen Menschen und der Natur Orientierung bieten, denn sie „leben bewusster mit sich und ihrer Umwelt und nachdem ich so was anstrebe, suche ich mir natürlich auch Leute, die das schon tun, die auch schon auf dem Weg sind, gerade um das vielleicht zu verstärken“" (1/4/31 ff.).

Wie bei TN 82 wird die bildungsbiografische Bedeutung des Engagements auch bei TN 72 erst durch die Beschreibung seiner Erwerbstätigkeitssituation in vollem Ausmaß sichtbar. Auch wenn TN 72 seine Stelle in der Datenverarbeitung gezielt in der Erwartung ausgesucht hatte, dass diese Arbeit nicht mit seinem ökologischen Denken in Konflikt stehe, erlebt er seine Erwerbsarbeitssituation dennoch als „das krasse Gegenteil“" (1/5/16) zu seinem Engagement im ökologischen Arbeitskreis, da dort kein Raum für ökologische Themen-, Frage- und Problemstellungen sei. Stattdessen wird er in seiner Wahrnehmung von den Kollegen als „Spinner angesehen“ (1/6/19), fühlt sich unterlegen und vermeidet Diskussionen. Er berichtet, beruflich "auch gerne was anderes machen" (1/5/18) zu wollen, jedoch bestehe aufgrund der schlechten Arbeitsmarktlage dazu keine Möglichkeit. Zudem schließt TN 72 anders als TN 82 - die berufliche Selbstständigkeit als Option aus, da er ,nicht so risikofreudig“" (1/5/21) sei. Die Intensität der Belastung durch den Berufsalltag und die berufliche Perspektivlosigkeit sowie die zentrale Bedeutung des Engagements in einer Gruppe Gleichgesinnter markiert TN 72 in der Aussage, ohne den Arbeitskreis „würde ich das wahrscheinlich nicht aushalten, in dem Beruf zu arbeiten" (1/5/17). Damit macht er auch indirekt deutlich, dass für ihn - anders als für TN 82 - eine Aufgabe des Engagements nicht infrage kommt, auch wenn er sich mittlerweile zudem in anderen Bereichen der ökologischen Community - z. B. in einer Lebensmittelkooperative - bewegt und engagiert.

Während TN 72 im Kontext seiner von ihm als in hohem Maße als psychisch belastend erlebten Erwerbsarbeit keinen Raum zur Selbstverwirklichung findet, bietet ihm das Engagement in der Ökologiebewegung einen Schonraum, in welchem es ihm in all seiner Verunsicherung möglich ist, authentisch aufzutreten, sich an den anderen 
Mitgliedern zu orientieren, sich mit dem für ihn identitätsbedeutsamen Streben nach einem besseren Umgang mit ökologischen Problemstellungen und einer ökologisch verantwortungsbewussten Lebensführung auseinanderzusetzen und letztlich Sicherheit im Umgang mit sich selbst, anderen Menschen und der Umwelt zu gewinnen. Das ökologische Engagement stellt für ihn zu diesem Zeitpunkt einen Ort dar, an dem er authentisch sein und zugleich eine stabile Identität aufbauen kann. Allerdings scheinen die Erfahrungen und Aneignungsprozesse im freiwilligen Engagement für TN 72 - im Gegensatz zum TN 82 - kein hohes Anregungspotenzial zur Erwägung umfassender beruflicher oder engagementbezogener Veränderungen zu beinhalten.

\subsection{TN 41: Freiwilliges Engagement als Ort des Anerkennungserlebens}

TN 41 ist zum Zeitpunkt des zweiten Interviews (2008) 65 Jahre alt, verheiratet und pensioniert. Sie entstammt einer Flüchtlingsfamilie, hat als Verwaltungswirtin seit den 1980er Jahren bei einer Bundesbehörde gearbeitet, berufsbegleitend eine Krankenhausseelsorgeausbildung sowie Suchtkrankenhelferausbildung absolviert, wurde danach die erste Suchtkrankenhilfe in der Behörde und bekleidete später bis zu ihrer Pensionierung dort die Stelle der Gleichstellungsbeauftragten. Vor ihrer Pensionierung hat TN 41 sich immer wieder in unterschiedlichen Formen freiwillig engagiert, aber erst nach ihrer Pensionierung steigert sich die Intensität ihres Engagements. Sie engagiert sich zum Zeitpunkt des Interviews besonders für Migrantenkinder/ -familien in der Nachbarschaft.

Auch bei TN 41 wird die bildungsbiografische Bedeutung ihres Engagements erst vor dem Hintergrund ihrer im Interview geschilderten Lebensgeschichte sowie der aktuellen Lebenssituation ersichtlich. TN 41 berichtet, eine ,ganz, ganz schlimme Kindheit" (I2/S. 38/Z.17) gehabt zu haben. Ihre Familie flüchtete 1952 nach Westdeutschland. Dort lebte die neunköpfige Familie in beengten Wohnverhältnissen und unter finanziell prekären Bedingungen. Als ältestes Kind musste sich TN 41 um die jüngeren Geschwister kümmern, da die Mutter ,immer depressiv“ (2/38/19) war und ihren erzieherischen Aufgaben nicht nachkommen konnte. Diese Verantwortungszumutung stellt eine Form der massiven Nicht-Anerkennung des Kindseins von TN 41 dar. Zudem erhielt sie auch für die Übernahme der erzieherischen Aufgaben ihrer Mutter keinerlei Anerkennung. Die Erfahrung der Nicht-Anerkennung machte sie aber nicht nur in der eigenen Familie, sondern auch im Wohnumfeld, denn dort wurden Flüchtlinge ,gehasst“ (2/24/45) und waren gewaltvollen Übergriffen ausgesetzt. Lichtblicke in ihrer Kindheit waren die Aufenthalte bei einer Familie in Skandinavien, denn dort erfuhr sie „praktisch richtig Familienanschluss“ (2/25/7) und machte darüber positive Anerkennungserfahrungen. Als Erwachsene wurde TN 41 von der früheren Nutznießerin des Engagements anderer selbst zur Engagierten für andere, indem sie vereinzelt über ein Austauschprogramm vermittelte Studenten aus anderen Ländern bei sich aufnahm, diese in die Stadt ,zu integrieren“ (2/42/24) und dabei auch etwas über deren Herkunftsländer zu erfahren versuchte. Dieses Interesse an ihren Gästen stellt eine Form der Anerkennung dar, welche TN 41 nicht in der eigenen Familie und dem Wohnumfeld, sondern lediglich in der skandinavischen Gastfamilie erlebte hatte. Sie erwähnt auch, sich zudem in der katholischen Nachbarschaftshilfe engagiert zu haben. Zwar geht sie auf dieses Engagement nicht näher ein, 
jedoch stellt die Engagementform des Helfens eine Form der gelebten Anerkennung für andere, zumeist benachteiligte, Menschen dar.

Dieser Einsatz für Partizipation und Anerkennung anderer Menschen, dessen Wurzeln sich bis in ihre Kindheit zurückverfolgen lassen, gewann auch im Kontext der Erwerbsarbeit als Verwaltungswirtin für TN 41 einen hohen Stellenwert. Sie stellte fest, dass es in der Bundesbehörde, bei der sie arbeitete, keine Sozialberaterin für die Angestellten gab, obwohl aus ihrer Sicht eine solche dringend notwendig gewesen wäre. In Absprache mit der Betriebsärztin absolvierte sie berufsbegleitend eine Krankenhausseelsorgeausbildung sowie eine Ausbildung zur Suchtkrankenhelferin. Anschließend bekleidete sie in ihrer Bundesbehörde zunächst den Posten der Suchtkrankenhilfe (hierfür wurde sie teilweise freigestellt) und bewarb sich später erfolgreich auf die ausgeschriebene Stelle als Gleichstellungsbeauftragte. In beiden Funktionen setzte sie sich - diesmal beruflich - für die Partizipation und Anerkennung von Menschen in schwierigen Lebenssituationen bzw. benachteiligte Personengruppen ein. Ihr kämpferisches Bemühen, die patriarchalen Strukturen der Behörde aufzubrechen und Diskriminierung von Frauen im Betrieb zu unterbinden, zog Konflikte mit den männlichen Führungskräften sowie dem Personalrat nach sich. Als Gleichstellungsbeauftragte nutzte sie eine damals bestehende Gesetzeslücke und stellte eine eigenständig Frauenliste auf, über welche die Frauen in sämtliche Gremien des Betriebs einzogen. Ihre Aktivitäten beschränkten sich nicht nur auf den Einsatz für Anerkennung und Partizipation der Frauen im eigenen Betrieb. Im Bewusstsein, dass ihre Möglichkeiten als einzelne Gleichstellungsbeauftragte im Betrieb begrenzt waren, erkannte sie: „Wir müssen uns verbünden“" $(2 / 15 / 21)$ und gründete einen übergreifenden Kreis, der den Gleichstellungsbeauftragten, die in ihren Betrieben allesamt „Einzelkämpferinnen“ (2/16/7) und „Außenseiter“ (2/16/10) waren, ein Gesprächs- und Austauschforum bot. Durch ihren beruflichen Einsatz ermöglichte TN 41 nicht nur anderen Frauen Anerkennung und Partizipation, sondern erfuhr auch selbst Anerkennung durch ihre Erfolge sowie die Nutznießerinnen ihres Engagements.

TN 41 schildert, dass ihre Arbeit als Gleichstellungsbeauftragte mit ,sehr viel Stress“ (2/4/28 f.) einherging und sie „viel viel Kraft“ (2/14/44) kostete. Dennoch sei sie nach der Pensionierung ,in " $n$ großes Loch gefallen“ (2/36/34 f.). Obwohl sie sich im Vorfeld darauf gefreut hatte, war das Rentnerinnendasein für sie zunächst „ganz schrecklich“ (2/36/35), da ,ich nicht mehr gebraucht wurde“ (2/36/35). Von Dauer war diese ,richtige Leere“ (2/36/38) allerdings nicht, denn sie fand im freiwilligen Engagement für Migrantenkinder und deren Familien eine Möglichkeit, sich weiterhin für die Anerkennung und Partizipation benachteiligter Personengruppen einzusetzen. Ursprünglich wollte sie nach einem Mädchen suchen, das sie unterstützen konnte, ,weil ich immer sage, Mädchen haben ja eh die Förderung noch viel nötiger. Ich will immer gezielt Frauen fördern" (2/7/49 f.). Aber bei dieser Suche lernte sie eine Mutter von zwei Jungen mit Migrationshintergrund kennen, die ihr von massiven Problemen mit der Institution Schule berichtete. TN 41 erkannte: „Die braucht ja eigentlich Hilfe, nicht, und hab mich dann auch eingeschaltet“ (2/8/15). Ihr Engagement für diese Familie fand auf mehreren Ebenen statt: Einerseits gab sie den Kindern Nachhilfe, andererseits unterstützte sie die Mutter im Umgang mit den Lehrern und erwirkte eine bessere Förderung der Kinder in der Schule. Letztlich hat sie durch ihr mehrjähriges Engagement für diese Kinder zu deren Schulerfolg beigetragen. Wie 
zuvor beruflich setzte TN 41 sich auch in ihrem freiwilligen Engagement erfolgreich und intensiv für Anerkennung und Partizipation benachteiligter Personengruppen ein und ermöglichte dabei nicht nur anderen Menschen Anerkennung, sondern erlebte auch selbst Anerkennung über die Erfolge ihres Engagements, die damit einhergehende Freude ihrer Schützlinge und die Wertschätzung von Integrationsvereinen. Dieses Engagement setzt TN 41 nach einem Umzug mit ihrem Ehemann auch in der neuen Nachbarschaft fort. Sie gibt nun dort den Migrantenkindern Nachhilfe, unternimmt mit ihnen Ausflüge und unterstützt Migrantenfamilien bei der Wohnungssuche oder im Umgang mit Ämtern. Dabei erfährt auch sie selbst Anerkennung durch die Reaktionen und Rückmeldungen ihrer Schützlinge. Zusammenfassend pointiert sie: „Ich äh gebe ja nicht nur, sondern ich krieg ja auch Freude zurück“ (2/38/1 f.).

Zukünftig will TN 41 nicht nur ihr Engagement fortsetzen, sondern erwägt zudem die Gründung einer multikulturellen Nachbarschaftshilfe, womit sie ähnlich wie schon als Gleichstellungs- und Frauenbeauftragte nicht nur das konkrete Engagement für die Benachteiligten, sondern auch die Vernetzung der Engagierten in den Blick nehmen würde.

Während TN 82 und TN 72 in ihren Erzählungen von einem zeitlich parallel zur Erwerbstätigkeit praktizierten Engagement berichten, welches ihnen die Realisierung ihres Strebens nach Autonomie bzw. Authentizität in einer Weise ermöglicht, die ihnen die Erwerbsarbeitssituation zu dieser Zeit nicht bietet, hat für TN 41 - auch wenn sie sich schon vor ihrer Pensionierung engagierte - das freiwillige Engagement erst nach der Beendigung ihrer Erwerbstätigkeit einen zentralen biografischen Stellenwert. Da sie bereits pensioniert ist, hat das freiwillige Engagement für TN 41 - anders als für TN 82 - nicht die bildungsbiografische Bedeutung eines Impulsgebers für mögliche berufliche Veränderungen. Vielmehr bietet ihr das Engagement für Migrantenkinder/-familien eine Möglichkeit, das durch das Wegbrechen der Erwerbsarbeit entstandene Vakuum auszufüllen, da sie sich weiterhin für Anerkennung und Partizipation benachteiligter Personengruppen einsetzen und über ihre Erfolge sowie die positiven Reaktionen Anerkennung erfahren kann. Die Aneignungsprozesse und Erfahrungen im Engagement haben für TN 41 keinen berufsbezogenen, jedoch durchaus einen engagementbezogenen Impulscharakter, da sie vor dem Hintergrund ihrer bisherigen Erfahrungen Überlegungen zur Erweiterung und Steigerung ihres freiwilligen Engagements anstellt.

Diese drei kurzen Fallskizzen zeigen auf, dass die bildungsbiografische Bedeutung des Engagements aus der Perspektive der Subjekte in einem dreipoligen Spannungsfeld zwischen den Polen Autonomie (TN 82), Authentizität (TN 72) und Anerkennung (TN 41) oszilliert und somit das Engagement einen Raum zur Realisierung identitätsrelevanter Orientierungen darstellt. Dabei kann das freiwillige Engagement zur Entwicklung bzw. zum Aufbau (TN 72), zur Fortsetzung bzw. Kontinuierung (TN 41) oder zur Modifikation und Transformation (TN 82) dieser Orientierung beitragen.

\section{Temporalität des freiwilligen Engagements und dessen subjektiver bildungsbiografischer Bedeutung}

Nachdem im vorangegangenen Kapitel das Spektrum der bildungsbiografischen Bedeutung des freiwilligen Engagements im Spannungsfeld von Autonomie, 
Authentizität und Anerkennung entfaltet wurde, soll im Folgenden unter Hinzuziehung des zweiten Interviews des Falls TN 82 exemplarisch dargestellt werden, dass sich sowohl freiwilliges Engagement als auch dessen bildungsbiografische Bedeutung über die Zeit hinweg wandeln.

TN 82 hatte im ersten Interview problematisiert, als angestellter Ingenieur kaum und auch im freiwilligen Engagement im ökologischen Arbeitskreis zu limitierte Möglichkeiten zum autonomen Handeln und Entscheiden zu haben und Überlegungen angestellt, durch Veränderungen in beiden Bereichen eine Steigerung seiner Handlungs- und Entscheidungsautonomie zu erreichen. Ob und wie TN 82 diese Überlegungen tatsächlich in der Zukunft in die Tat umgesetzt hat, kann erst durch den Blick auf das zweite Interview (2009) beantwortet werden. Dort berichtet er, sich schon Mitte der 1980er Jahre selbstständig gemacht zu haben und nunmehr auf eine 25-jährige - sich über verschiedene Stationen erstreckende - berufliche Selbstständigkeit zurückzublicken. Zunächst arbeitete er kurzzeitig freiberuflich als IT-Berater, gründete aber bald mit Gleichgesinnten eine Genossenschaft als „selbst verwalteten Betrieb“ (2/1/13 f.), welche - aus Sicht von TN 82 aufgrund des mangelnden Einsatzes der Mitbegründer - nach kurzer Zeit geschlossen wurde. Danach war er erneut freiberuflich tätig, bis er Mitte der 1990er Jahre eine Internetproviderfirma gründete, welche er später aufgrund der großen Konkurrenz verkaufte. Seitdem arbeitet TN 82 als „Einzelkämpfer“ (2/1/10) bzw. freiberuflicher IT-Berater. Er vermutet, die Selbstständigkeit lag ihm aufgrund der beruflichen Selbstständigkeit seines Vaters „schon wahrscheinlich ein bisschen im Blut" (2/2/9).

Verändert hat sich zwischen 1984 und 2009 nicht nur die Berufssituation, sondern auch das freiwillige Engagement von TN 82. Hatte er 1984 in sein Engagement in der Ökologie- und Friedensbewegung noch viel Energie investiert und sogar die Arbeitszeit reduziert, damit „ich für meine anderen Aktivitäten auch Zeit hatte“ (2/7/1 f.), so engagiert er sich 2009 nicht mehr. Auch wenn er in seinen Erzählungen nur vereinzelt auf sein früheres ökologisches Engagement eingeht, so resümiert er rückblickend ,das war schon sehr entscheidend für mich, weil es mich schon sehr geprägt hat auch, und war für mich eine wichtige Zeit damals" (2/4/17 f.). Wann und warum er sein Engagement in der Ökologie- und Friedensbewegung aufgab, berichtet er nicht, jedoch deutet er an, dass Selbstständigkeit, Heirat und Familiengründung keine zeitlichen Ressourcen für ein intensives Freizeitengagement ließen. Zudem verweist er darauf, dass das Thema Ökologie zwar in den 1980er Jahren noch „ziemlich neu“ (2/4/17) gewesen sei, mittlerweile aber ,ist das Wort Ökologie in jedem Wort, in jeder Werbung kommt es vor, überall“ (2/4/15 f.). Damit verweist er einerseits auf den Erfolg der ökologischen Aufklärung, andererseits aber auch darauf, dass im Zuge dieses Erfolges die ökologische Aufklärung als Engagementfeld ihren ehemals innovativen Charakter verloren hat, welcher für TN 82 neben der Möglichkeit zum autonomen Handeln einen Anreiz zum ökologischen Engagement dargestellt hatte. In den 1990er Jahren engagierte TN 82 sich noch einmal zeitweise in der damals neu aufkommenden Bürgernetzbewegung. Auf dieses Engagement geht er nur marginal ein, berichtet aber, dass es sich - im Gegensatz zum ökologischen Engagement - an der Schnittstelle von Berufstätigkeit und Engagement bewegte. Beispielsweise sei beim Engagement auch der Kontakt zu seinem gegenwärtigen Hauptklienten zustande gekommen. Nach diesem Engagement engagierte er sich nur 
noch einmal punktuell, als er um 2000 für den Kindergarten, den sein Kind besuchte, die Homepage aufbaute.

Beim Blick auf beide Interviews zeigt sich der Aspekt der Temporalität des freiwilligen Engagements sowie der biografischen Bedeutung des Engagements. 1984 war das Engagement in der Ökologie- und Friedensbewegung für TN 82 ein zentraler Möglichkeitsraum zum autonomen Handeln, welches ihm im Kontext der Erwerbsarbeit damals nicht möglich war. Die Temporalität seines Engagements deutete sich schon im ersten Interview in seinen Überlegungen zur Suche nach Engagementformen/-bereichen an, welche ihm die weitere Steigerung des autonomen Handelns ermöglichen. Aber wohl erst im Zuge der Realisierung der beruflichen Neuorientierung kam es zur Aufgabe des ökologischen Engagements. Die Autonomieerfahrungen und Aneignungsprozesse im freiwilligen Engagement wurden somit zum Impulsgeber für eine faktische berufliche Veränderung, in deren Folge das bisherige Engagement aufgegeben wurde. ${ }^{7}$ Denn mit dem Schritt in die berufliche Selbstständigkeit verlor das bisherige Engagement für TN 82 sein Alleinstellungsmerkmal als Möglichkeitsraum des autonomen Handelns und damit als bedeutsame Gegenwelt zur Erwerbsarbeit und wurde nicht fortgesetzt. Das spätere Engagement in der Bürgernetzbewegung an der Schnittstelle von Engagement und Erwerbsarbeit sowie das punktuelle Engagement für den Kindergarten hatten für TN 82 nicht mehr den Charakter einer positiven Gegenwelt gegenüber der Erwerbsarbeit und damit nicht mehr die bildungsbiografische Relevanz als primärer Ort des Autonomieerlebens und der Selbstbestimmung.

\section{Kontextualität des freiwilligen Engagements}

Auch wenn die bisherigen Ausführungen auf die bildungsbiografischen Bedeutungen des Engagements und deren Temporalität abzielten, deutete sich bereits an, dass freiwilliges Engagement und dessen subjektive Bedeutung weder ausschließlich über die individuellen noch ausschließlich über die kollektiven Kontexte, sondern nur über die Berücksichtigung ihrer wechselseitigen Verwobenheit erschlossen werden können. Die Verwobenheit dieser Kontexte zeigt sich eindrücklich am Fall von TN 82: In den 1980er Jahren kamen neue soziale Bewegungen wie die Friedens-, Ökologie- und Anti-Atomkraftbewegung aus dem Protest gegen bestehende Strukturen auf. Ökologische Aufklärung wurde langsam zu einem gesellschaftspolitischen sowie pädagogischen Thema. Zudem entstanden im Zuge der Hinwendung zur Adressatenorientierung in den Institutionen der Erwachsenenbildung neuartige Settings. Der an die VHS angebundene ökologische Arbeitskreis kann somit als Ausdruck der in den 1980er Jahren aktuellen pädagogischen und gesellschaftlichen Diskussions- und Erkenntnisstände betrachtet werden. Das ökologische Interesse von TN 82 geht mit

\footnotetext{
${ }^{7}$ Auch TN 72 hat - obwohl er im ersten Interview berichtete, keine Perspektiven für eine berufliche Veränderung zu sehen - seine unbefriedigende und belastende Erwerbsarbeit zeitnah nach dem ersten Interview aufgegeben und in der Folgezeit u. a. in der Ökobranche gejobbt und sich später gezielt für eine Stelle an einem Umweltinstitut entschieden. Auch hier zeigt sich, dass sich die im Engagement gesammelten Erfahrungen und Aneignungsprozesse in einer Art und Weise auf andere Lebensbereiche auswirken können, die zu einem bestimmten Zeitpunkt vom Engagierten noch gar nicht abgesehen werden können.
} 
der beginnenden öffentlichen Diskussion ökologischer Themen einher. All dieses sind kollektive Kontexte, welche das Feld des ökologischen Engagements in den 1980er Jahren prägen und strukturieren. Aber der Fall von TN 82 macht deutlich, dass die Entscheidung für ein ökologisches Engagement im Allgemeinen und das Engagement im ökologischen Arbeitskreis im Speziellen keineswegs nur im Rückgriff auf die kollektiven Kontexte, sondern erst durch die zusätzliche Berücksichtigung der individuellen Kontexte wie der Lebensgeschichte und der aktuellen Lebenssituation erklärbar wird. Erst vor dem Hintergrund seiner Suche nach Möglichkeiten zum autonomen Handeln, des Fehlens dieser Möglichkeiten im Rahmen der Erwerbsarbeitssituation, der Erkenntnis der Notwendigkeit des ökologischen Engagements sowie der Entdeckung der sich ihm im ökologischen Arbeitskreis bietenden Möglichkeiten zum autonomen Handeln wird nachvollziehbar, warum TN 82 für diese Form des Engagements zugänglich war. Zugleich wird auch die Aufgabe des ökologischen Engagements erst durch die mit dem Schritt in die Selbstständigkeit vollzogene berufliche Veränderung verständlich.

Freiwilliges Engagement ist demnach in ein komplexes Kontextgefüge eingebettet, welches sich wie folgt systematisieren lässt: $\mathrm{Zu}$ den individuellen Kontexten zählen erstens die auf bisherigen Lebenserfahrungen basierende, aktuelle bildungsbiografische Orientierung im Spannungsfeld von Autonomie, Authentizität und Anerkennung, zweitens die Einschätzung, ob und inwieweit die Realisierung dieser Orientierung in anderen bedeutsamen Lebensweltbezügen möglich ist, drittens die jeweils aktuelle Lebenssituation (insbesondere die Zeit- und Kraftressourcen) sowie viertens das Erkennen des Engagements als Möglichkeitsraum zur Realisierung der aktuellen bildungsbiografischen Orientierung. $\mathrm{Zu}$ den kollektiven Kontexten zählen erstens die gesellschaftlich institutionalisierte Strukturierung des Lebenslaufs (insbesondere Übergänge wie die Verrentung), zweitens die aktuellen gesellschaftlichen, pädagogischen und politischen Diskurse, durch welche die Subjekte für Inhalte/Probleme sensibilisiert werden, für die sich ein Engagement ihrer Einschätzung nach lohnt, drittens die häufig mit den Diskursen verwobenen aktuellen politischen, gesellschaftlichen und globalen Ereignisse, Entwicklungen und Entscheidungen, viertens die sich neu formierenden politischen oder sozialen Gruppierungen und fünftens die aktuellen pädagogischen Entwicklungen, Konzepte, Settings und Institutionalformen.

\section{Freiwilliges Engagement - ein dynamisch angelegter und temporär genutzter Erfahrungs- und Bildungsraum}

Freiwilliges Engagement als Erfahrungsraum ermöglicht den Engagierten bildungsbiografisch bedeutsame Erfahrungen und Aneignungsprozesse im Spannungsfeld von Autonomie, Authentizität und Anerkennung, welche ihnen in anderen Lebensweltbezügen nicht oder zumindest nicht in dieser Form möglich sind. Diese Aneignungs- und Bildungsprozesse können ihrerseits durchaus Auswirkungen auf andere Lebensbereiche (z. B. Veränderungen in der Erwerbsarbeit) der Engagierten haben, mittelfristig zur Veränderung ihres Engagements führen oder sogar die Aufgabe des Engagements nach sich ziehen. 
Freiwilliges Engagement als Erfahrungs- und Bildungsraum zeichnet sich aufgrund seiner Eingebundenheit in je aktuelle gesellschaftliche, politische und pädagogische Ereignisse und Diskurse durch seine auf Dynamik und Temporalität hin ausgerichtete Gesamtstruktur aus. Kollektive Entwicklungen führen zur Entstehung und Etablierung neuer Felder, Themen, Formen und Settings freiwilligen Engagements oder können dazu beitragen, dass bisher noch eher marginal bekannte Bereiche, Inhalte oder Praktiken einen Bedeutungs- und Engagiertenzuwachs bekommen. Auf diese Weise vergrößert sich das Spektrum an Engagementformen, Engagementthemen und Engagementsettings, aus welchen sich Menschen das für sie passende Engagement auswählen können. Es kann aber im Zuge gesellschaftlicher Entwicklungen auch umgekehrt zu einer Marginalisierung langjährig etablierter oder kurzfristig gefragter Engagementbereiche oder Engagementfelder kommen, was potenziell Engagierten den Zugang erschweren kann. Ein Beispiel für die Temporalität von Engagementformen und Engagementfeldern, welche wiederum zu der starken Dynamik des freiwilligen Engagements in seiner Gesamtheit führt, stellt der an die VHS angegliederte Arbeitskreis mit dem Ziel der ökologischen Aufklärung der Öffentlichkeit dar. Das ökologische Engagement im Arbeitskreis ist hinsichtlich seiner inhaltlichen Ausrichtung sowie des Settings ein Ausdruck der in den 1980er Jahren (wieder) erblühenden ökologischen Aufklärungsbewegung sowie der damaligen Abkehr von der früheren Leiterzentrierung in der Erwachsenenbildung. 25 Jahre später stellt diese Art der ökologischen Erstaufklärung kein derartig zentrales und innovatives Engagementthema und Engagementsetting mehr dar, da mittlerweile eine Veralltäglichung und Verselbstverständlichung ökologischer Themen stattgefunden hat. Aber nicht nur das Spektrum der Engagementformen und Engagementthemen ändert sich stetig, sondern auch die Engagierten, deren Lebensweltbezüge und somit auch deren Engagement unterliegen einem ständigen Wandel. Somit weist das freiwillige Engagement aufgrund seiner sich stetig wandelnden Gesamtarchitektur und der Temporalität des individuellen Engagements jedes Engagierten eine doppelte dynamische Struktur auf. Der Erfahrungsraum des freiwilligen Engagements stellt somit für die Engagierten eine „raum-zeitlich erfahrbare soziale Welt“ (Kade 1993, S. 398) dar, in welcher sie je (gegenwarts-)spezifische Bildungserfahrungen sammeln sowie Bildungsprozesse erleben können.

Open Access Dieser Artikel unterliegt den Bedingungen der Creative Commons Attribution License. Dadurch sind die Nutzung, Verteilung, und Reproduktion erlaubt, sofern der/die Originalautor/en und die Quelle angegeben sind.

\section{Literatur}

Braun, S. (2000). Bürgerschaftliches Engagement - Konjunktur und Ambivalenz einer gesellschaftlichen Debatte. Leviathan. Zeitschrift für Sozialwissenschaft, 1, 83-109.

Düx, W., et al. (2008). Kompetenzerwerb im freiwilligen Engagement. Eine empirische Studie zum informellen Lernen im Jugendalter. Wiesbaden: VS Verlag.

Enquete-Kommission „Zukunft des Bürgerschaftlichen Engagements“ des Deutschen Bundestages. (2002). Bericht. Bürgerschaftliches Engagement: auf dem Weg in eine zukunftsfähige Bürgergesellschaft (Bd. 4). Opladen: Leske + Budrich. 
Fischer, M., \& Kade, J. (2012). Qualitative Längsschnittstudien in der Erwachsenen- und Weiterbildungsforschung. In B. Schäffer \& O. Dörner (Hrsg.), Handbuch Qualitative Erwachsenen- und Weiterbildungsforschung (S. 612-620). Opladen: Verlag Barbara Budrich.

Fischer, M., Kade, J., \& Benedetti, S. (2010). Chronographien - Bildungsbiographische Bewegungen im Raum. In R. Egger \& B. Hackl (Hrsg.), Sinnliche Bildung? Pädagogische Prozesse zwischen vorprädikativer Situierung und reflexivem Anspruch (S. 253-268). Wiesbaden: VS Verlag.

Gensicke, T., Picot, S., \& Geiss, S. (2006). Freiwilliges Engagement in Deutschland 1999-2004. Ergebnisse der repräsentativen Trenderhebung zu Ehrenamt, Freiwilligenarbeit und bürgerschaftlichem Engagement. Wiesbaden: VS Verlag.

Hansen, S. (2008). Lernen durch freiwilliges Engagement. Eine empirische Studie zu Lernprozessen in Vereinen. Wiesbaden: VS Verlag.

Hof, C., Kade, J., \& Fischer, M. (2010). Serielle Bildungsbiographien. Auf dem Weg zu einem qualitativen Bildungspanel zum Lebenslangen Lernen. Zeitschrift für Pädagogik, 3, 328-339.

Honneth, A. (1992). Kampf um Anerkennung. Zur moralischen Grammatik sozialer Konflikte. Frankfurt a. M.: Suhrkamp Verlag.

Hübner, A. (2010). Freiwilliges Engagement als Lern- und Entwicklungsraum. Eine qualitative empirische Studie im Feld der Stadtranderholungsmaßnamen. Wiesbaden: VS Verlag.

Humboldt, W. von. (1960). Theorie der Bildung des Menschen. Bruchstück [1793]. In A. Flitner \& K. Giel (Hrsg.), Wilhelm von Humboldt, Werke in fünf Bänden, Bd. I.: Schriften zur Anthropologie und Geschichte (S. 234-240). Stuttgart: Metzler Verlag.

Kade, J. (1993). Aneignungsverhältnisse diesseits und jenseits der Erwachsenenbildung. Zeitschrift für Pädagogik, 3, 391-408.

Kade, J. (2012). Bildungstheorie und Bildungsforschung. In B. Schäffer \& O. Dörner (Hrsg.), Handbuch Qualitative Erwachsenen- und Weiterbildungsforschung (S. 37-49). Opladen: Verlag Barbara Budrich.

Kade, J., \& Hof, C. (2010). Die Zeit in der (erziehungswissenschaftlichen) Biographieforschung. Theoretische, methodologische und empirische Aspekte ihrer Fortschreibung. In J. Ecarius \& B. Schäffer (Hrsg.), Typenbildung und Theoriegenerierung. Methoden und Methodologien qualitativer Bildungsund Biographieforschung (S. 145-167). Opladen: Verlag Barbara Budrich.

Kade, J., Hof, C., \& Peterhoff, D. (2008). Verzeitlichte Bildungsgestalten: Subjektbildung im Kontext des Lebenslangen Lernens. Report. Zeitschrift für Weiterbildungsforschung, 4, 9-22.

Jakob, G. (2001). Wenn Engagement zur „Arbeit“ wird ... - Zur aktuellen Diskussion um freiwilliges Engagement im Wandel der Arbeitsgesellschaft. In R. Heinze \& T. Olk (Hrsg.), Bürgerengagement in Deutschland. Bestandsaufnahme und Perspektiven (S. 167-188). Opladen: Leske+Budrich.

Luhmann, N. (2002). Das Erziehungssystem der Gesellschaft. Frankfurt a. M.: Suhrkamp Verlag.

Nohl, A.-M. (2008). Interview und dokumentarische Methode. Anleitungen für die Forschungspraxis (2. überarb. Aufl.). Wiesbaden: VS Verlag.

Olk, T., \& Hartnuß, B. (Hrsg.). (2011). Handbuch Bürgerschaftliches Engagement. Weinheim: Beltz Juventa Verlag.

Priller, E. (2010). Stichwort: Vom Ehrenamt zum zivilgesellschaftlichen Engagement. Zeitschrift für Erziehungswissenschaft, 13, 195-213.

Rosenbladt, B. von (Hrsg.). (2001). Freiwilliges Engagement in Deutschland. Freiwilligensurvey 1999. Ergebnisse der Repräsentativerhebung zu Ehrenamt, Freiwilligenarbeit und bürgerschaftlichem Engagement (Bd.) 1: Gesamtbericht (2. korr. Aufl.). Stuttgart: Verlag W. Kohlhammer.

Söker, R., \& Mutz, G. (2003). Lernen in Tätigkeitsfeldern bürgerschaftlichen Engagements - Transferprozesse in die Erwerbsarbeit. Fallstudien in ausgewählten Regionen Deutschlands. Quem-Materialien 46. Berlin. http://www.abwf.de/content/main/publik/materialien/materialien46.pdf. Zugegriffen: 3. April 2014.

Strauss, A. (1991). Grundlagen qualitativer Sozialforschung. Datenanalyse und Theoriebildung in der empirischen soziologischen Forschung. München: Wilhelm Fink Verlag.

Zeitschrift für Erziehungswissenschaft, Schwerpunkt. (2010) Zivilgesellschaft und freiwilliges Engagement ( Jg. 13, Heft 2). Wiesbaden: VS Verlag für Sozialwissenschaften. 